\title{
Medien in der Lehrerbildung
}

\author{
* Zurück zur Übersicht über den \\ Themenschwerpunkt
}

\section{\begin{tabular}{l} 
2 \\
\cline { 3 - 3 }
\end{tabular} dung Lehramtsstudierender}

\section{MICHAEL LIPPSTREU}

Bevor die eigentlichen Ausführungen beginnen können, ist es nötig, auf ein bereits im Titel anklingendes Grundproblem des folgenden Artikels einzugehen. Dieses entsteht, da sich der Bildungsbegriff hier als problematisch erweist. Die Problematik resultiert aus dem Umstand, dass Bildung an sich individueller Selbstzweck ist, während die Bildung angehender Lehrerinnen und Lehrer sich als gesellschaftlicher Selbstzweck erweist. Synergieeffekte sind hierbei glücklicherweise unvermeidlich! Für den Kontext der Lehrerbildung bedeutet dies in letzter Konsequenz, dass sich an den Ruf nach einer Mediengrundbildung Lehramtsstudierender stets der Wunsch knüpft, diese mögen ihrerseits für eine ausreichende Mediengrundbildung der nächsten Generation sorgen. Es geht um (gewünschte) Handlungskompetenzen und Einstellungen (gegenüber Medien) der zukünftigen Lehrerinnen und Lehrer, um den Wunsch also, dass diese die neuen Medien kompetent in ihrem Unterricht einsetzen werden. Dieser Ansatz weicht von einem Bildungsgedanken, welcher Bildung als Selbstzweck versteht, ab. Die Frage, mit der sich der folgende Artikel also beschäftigt, lautet:

Wie ist es zu anzustellen, dass zukünftige Lehrerinnen und Lehrer mit einer positiven Selbstwirksamkeitserwartung bezüglich neuer Medien ins Berufsleben starten und dass sie die (neuen) Medien überhaupt in ihrem Unterricht einsetzen?

Diese Frage mag auf den ersten Blick als unzulässige Reduktion auf den Werkzeugcharakter des Mediums erscheinen. Im Folgenden soll aufgezeigt werden, warum sie als grundlegend angesehen werden muss und weshalb ebenfalls wichtige Fragen, wie die nach einem sinnvollen, reflektierten und verantwortungsbewussten Medienumgang in dem hier beschriebenen Kontext, als sekundär betrachtet werden können.

Das Medium sei die Botschaft, postulierte Marshall McLuhan 1964 in Understanding Media. Er machte darauf aufmerksam, dass bei der Analyse von Medien(-wirkungen) sehr genau zwischen dem Medium und dem transportierten Inhalt unterschieden werden muss. Die eigentliche Medienwirkung, so McLuhans Idee, sei völlig unabhängig vom Inhalt (vgl. McLuhan 1964). Diese Betrachtungsweise erscheint mir auch im Kontext der Konzeption einer für alle Lehramtsstudierenden verbindlichen Mediengrundbildung in der 1. Phase der Lehrerbildung für grundsätzlich richtig.

Übertragen auf diese Aufgabe wird daher eine konsequente Binomisierung vorgeschlagen. Der Medieneinsatz an sich und die Medienkompetenz, als Vermögen „zur sinnvollen, reflektierten und verantwortungsbewussten Nutzung der Medien" (Medienpädagogische Einrichtungen 2009, S. 1) müssen, wenn Nachhaltigkeit das Ziel der Lehrerbildung sein soll, konsequent getrennt betrachtet werden. Der Umstand, dass erst beide Elemente vereint die Medienbildung eines/r Lehrers/Lehrerin ausmachen, kann an dieser Stelle nicht weiter ausgeführt werden. Von Interesse ist zunächst, und darum wird es im Folgenden gehen, die Klärung des Verhältnisses beider zueinander.

Im Medienpädagogischen Manifest lässt sich nachlesen:

„Es gibt eine Fülle an hervorragenden medienpädagogischen Materialien für die Praxis, eine Vielzabl an überzeugenden Modellversuchen und eindrucksvollen Leuchtturmprojekten aber es feblt an der erforderlichen Nachbaltigkeit. Es mangelt nach wie vor an der Infrastruktur und an den organisatorischen Rahmenbedingungen in den Bildungseinrichtungen sowie an der medienpädagogischen Qualifikation der pädagogischen Fachkräfte" (Medienpädagogische Einrichtungen 2009, S. 1).

Einer Fülle gut dokumentierter und pädagogisch konzipierter Materialien steht demnach deren unzureichende Nutzung gegenüber. Die angeführten Gründe für dieses Missverhältnis müssen allerdings genauer hinterfragt werden. Ein Blick auf die materielle Ausstattung der Schulen zeigt, dass hier nicht von einer Mangelsituation als Hauptursache ausgegangen werden kann. Im IT-Bericht des Bundesministeriums für Bildung und Forschung aus dem Jahr 2006 finden sich folgende Angaben: 
„Die Relation Schüler/Schülerinnen pro Computer hat sich im vergangenen Jahr in den allgemein bildenden Schulen nochmals verbessert. In den Grundschulen teilen sich 12 Schülerinnen/ Schüler einen Computer. Auch in den Sekundarschulen I und II konnte die Relation Schülerinnen/Schüler pro Computer wieder gesteigert werden auf jetzt $11: 1$. ... $99 \%$ der bundesdeutschen Schulen sind mit stationären und mobilen Computern für den Unterrichtseinsatz, ausgestattet. ... In allen Schulformen ist weiterbin ein deutlicher Rückgang von ISDN-Anscblüssen zu verzeichnen. ... Über einen DSL-Anscbluss verfügen $66 \%$ der Sekundarschulen I und II, über eine Standleitung $11 \%$ " BMBF 2006, S. Gff.).

Für die Softwareausstattung der Sekundarstufen I und II finden sich neben anderen die folgenden Angaben: Lernsoftware (91 \%), Programme zum Erstellen multimedialer Anwendungen $(71 \%)$, Software mit Werkzeugcharakter $(51 \%)$ und Multimediale Nachschlagewerke (75\%) (vgl. BMBF 2006). Hinzu kommt, dass sich für eine Vielzahl aktueller Anforderungen, wenn entsprechende Programme nicht schon vom Hersteller mitgeliefert werden, leistungsstarke Freeware-Lösungen finden lassen. Es kann also unterstellt werden, dass die Ausstattung der Schulen mit Hard- und Software nicht der entscheidende Grund für die beklagte Situation ist.

Über die organisatorischen Rahmenbedingungen lässt sich an dieser Stelle lediglich festhalten, dass das Prozedere der Raumreservierung schulintern geregelt ist. Da im Zitat aber von einer mangelhaften Nutzung der Infrastruktur ausgegangen wird, scheint es eher unwahrscheinlich, dass Lehrerinnen und Lehrer vor ernsthafte Probleme gestellt werden, wenn sie sich für eine Stunde oder Einheit Computerarbeitsplätze reservieren wollen.

Als Stellgröße bleibt also die medienpädagogische Qualifikation der Lehrkräfte, dies ist insofern erfreulich, als dass dies zugleich der einzige der genannten drei Faktoren ist, auf den die Hochschulen unmittelbar einwirken können. Dass die mit der Bildung zukünftiger Lehrerinnen und Lehrer betrauten Hochschulen sich dieser Problematik bewusst sind und an Lösungen arbeiten, beweist nicht zuletzt das 2009 veröffentlichte Medienpädagogische Manifest.

Mit dem Vorschlag der getrennten Betrachtung von Medieneinsatz und Medienkompetenz im Rahmen des Lehramtsstudiums wird nun der Versuch unternommen die Problematik der medienpädagogischen Qualifikation Lehramtsstudierender ins Zentrum der Überlegungen zu rücken und einen erfolgversprechenden Lösungsweg zu skizzieren.
Als grundlegend problematisch erweist sich in diesem Zusammenhang zunächst der Umstand, dass sich für „die Bundesrepublik Deutschland ... ein generelles Defizit an lehrerbildungsbezogener empirischer Forschung feststellen“ (Blömeke 2004, S. 61) lässt. Aussagen hinsichtlich der zu erwartenden Wirksamkeit einzelner Vorschläge zur Verbesserung der Mediengrundbildung Lehramtsstudierender sind daher mit Bedacht vorzubringen. Will man dennoch einen Versuch wagen, so kommt man nicht umhin auf Kochs Längsschnittsuntersuchung von $1972 \mathrm{zu}$ verweisen. Koch kommt zu dem Ergebnis, dass ,vor dem Studium eher konservative Einstellungen vorhanden sind, während des Studiums liberalere Auffassungen stärkere $\mathrm{Zu}$ stimmung finden ..., bevor im Referendariat wieder zu konservativen Positionen zurückgekehrt wird“ (Blömeke 2004, S. 65ff.). Dieses Ergebnis legt den Schluss nahe, dass der Transfer theoretischer Studieninhalte in die Praxis oftmals nicht gelingt.

Auf der Suche nach einer möglichen Begründung für diesen Umstand stößt man auf den Begriff der beliefs. Dieser bezieht sich auf die „Vorannahmen, Einstellungen bzw. Erwartungen ... sowie Berufswahlmotive" (Blömeke 2004, S. 64) Lehramtsstudierender. Zentrale empirische Forschungsergebnisse zu beliefs legen nahe, dass diese erfahrungsbasiert und weitgehend veränderungsresistent sind und als Informationsfilter beschrieben werden können. Lernprozesse können vor allem dann erfolgreich sein, wenn sie produktiv an bestehenden beliefs anknüpfen (vgl. Blömeke 2004). Ausgehend von diesen Befunden kann unterstellt werden, dass tatsächlich erlebter Unterricht einen wesentlich größeren und anhaltenden Einfluss auf die zukünftige Unterrichtsgestaltung angehender Lehrerinnen und Lehrer hat als theoretische Studieninhalte. Diese Feststellung führt zur zentralen Aussage bezüglich der Unterstützung der Mediengrundbildung Lehramtsstudierender:

Die nachhaltige Förderung der Mediengrundbildung von Lehramtsstudierenden erfordert verbindliche e-learning-Angebote in allen Fachbereichen.

Gemäß der oben vorgeschlagenen getrennten Betrachtung von Mediennutzung und Medienkompetenz ist diese Forderung so zu verstehen, dass die Studienordnung einen festen Anteil an Leistungsnachweisen in e-learning ${ }^{-1}$-Veranstaltungen für alle Lehramtsstudierenden verbindlich vorschreibt. Der jeweilige fachwissenschaftliche oder fachdidaktische Inhalt der Veranstaltung ist dabei sekundär. Nur so kann gewährleistet werden, dass das Erleben von selbstverständlichem Medieneinsatz in der Lehre kein Theoretikum bleibt. Sinnvoll erscheint es darüber hinaus Schulpraktika, wie sie an den Pädagogischen Hochschulen Baden-Württembergs 
durchgeführt werden, um ein Praktikum zum Thema „Lehren und Lernen mit Multimedia und Internet / e-learning“ zu erweitern. In diesem Praktikum müssten die Lehramtsstudierenden e-learning-Schulstunden konzipieren, umsetzen und reflektieren. So würde erreicht, dass sie e-learning-Angebote sowohl aus der Position der Lernenden als auch aus der der Lehrenden kennenlernen. Das erklärte Ziel einer solch grundlegenden Richtungsentscheidung für das Lehramtsstudium ist eine feste Verankerung des Lernens mit neuen Medien in der Bildungsbiografie Lehramtsstudierender.

Ausgehend von den oben beschriebenen Forschungsergebnissen zur Lehrerbildung kann davon ausgegangen werden, dass auf diesem Weg nachhaltig Handlungskompetenz aufgebaut werden kann. Auf dieser Grundlage sollte sich das ebenfalls oben benannte Defizit in der Nutzung der bereits vorhandenen Medieninfrastruktur an den Schulen relativieren.

Es ist ein Irrtum davon auszugehen, einige verbindliche Lehrveranstaltungen mit dem Ziel der theoriebasierten Medienbildung Lehramtsstudierender könnten vergleichbare Resultate in Aussicht stellen. Trotzdem müssen auch derartige Lehrveranstaltungen fester Bestandteil des Lehramtsstudiums sein. Sie sollen dafür sorgen, dass die Reflexion des Erlebten nicht der Beliebigkeit preisgegeben wird. Aufgabe des Fachbereichs Medienpädagogik in diesem Konzept ist die Entwicklung medienpädagogischer Materialien, der Austausch mit den einzelnen Fachdidaktiken und vor allem die Reflexion des Medieneinsatzes als wichtiger Teil der Medienbildung Lehramtsstudierender. Der Fachbereich Medienpädagogik muss Schalt-, Koordinationsund Reflexionszentrale für Lehrende und Studierende bei Fragen zur Gestaltung und Bewertung von e-learning-Angeboten sein.

Die getrennte Betrachtung von Mediennutzung und Medienkompetenz will nicht das Gewicht der Medienkompetenz im eingangs definierten Sinne anzweifeln. Sie dient vielmehr dazu aufzuzeigen, dass Medienkompetenz im Rahmen eines Lehramtsstudiums kein individueller Selbstzweck ist und daher Erfahrungen der selbstverständlichen Mediennutzung im Rahmen der eigenen Bildungsbiografie zur Voraussetzung hat. Ziel ist nicht der/die mediengebildete Lehrer/-in, sondern der kompetente Einsatz neuer Medien im Unterricht. Zugespitzt lässt sich festhalten, dass die Reflexion über die Mediennutzung als Merkmal von Medienkompetenz für das Ziel einer Steigerung des Einsatzes neuer Medien im Schulalltag sekundär ist. Vielmehr muss die Hochschule bildungsbiografisch erlebbar machen, was von den zukünftigen Lehrerinnen und Lehrern erwartet wird. Dazu genügt es nicht, dass Kopiervorlagen künftig statt in einem Ordner bei der Sekretärin auf Moodle oder ähnlichen Plattformen bereitgestellt werden oder dass im Seminar nicht mehr Folien und Tageslichtprojektor, sondern Laptop und Videoprojektor im Einsatz sind. Lehramtsstudierende müssen die Vorteile interaktiver, adaptiver, multicodaler, multimodaler und multimedialer Lernumgebungen ebenso aktiv kennenlernen wie die Vorteile voll- oder teilvirtueller e-learning-Angebote. Erst wenn in den Lehrveranstaltungen tatsächlich recherchiert, gechattet, geblogt, erstellt oder publiziert wird, besteht die Chance, dass diese Möglichkeiten der Unterrichtsgestaltung auch in der Schule keine extravaganten Experimente bleiben. Derzeit erscheint es noch so, als ob derartige Lehrangebote an der Hochschule gerne auf sich selbst referenzieren. So existieren e-learningSeminare zum Thema Lehren und Lernen mit neuen Medien, Weblogs werden eingesetzt, um etwas über die didaktischen Möglichkeiten von Weblogs zu lehren bzw. lernen, und in Foren wird darüber diskutiert, ob sich Foren eignen, um Diskussionen anzuregen. Dies unterstreicht eher den Sonderstatus von Multimedia und elearning, als zu dessen Selbstverständlichkeit beizutragen, und legt den Verdacht nahe, dass es an der Hochschule entweder erhebliche Ressentiments gegenüber dem Einsatz neuer Medien in Lehrveranstaltungen gibt oder dass es an hinreichend dokumentierten Szenarien für die Hochschullehre mangelt. Unabhängig davon, wie die Gemengelage hier im Detail aussieht, muss an dieser Stelle dezidiert für Weiterbildungsangebote an Hochschullehrende plädiert werden. Die Konzeption und Durchführung dieser Angebote soll vom Fachbereich Medienpädagogik übernommen werden, wobei dieser auf Anregungen und Fragen aus den Fächern angewiesen sein wird. Ebenso wie Lehrerinnen und Lehrer haben auch Hochschullehrende individuelle Vorstellungen davon, wie Lehre auszusehen hat. Diese Vorstellungen sind ebenfalls durch beliefs geprägt und lassen sich nicht wegverordnen. Hier ist Unterstützung und Anregung geboten, wenn die Integration von elearning-Angeboten in die Hochschullehre erfolgreich verlaufen soll.

Die Überlegung, der Wunsch nach einer Mediengrundbildung für angehende Lehrerinnen und Lehrer impliziere, dass diese in ihrem späteren Berufsleben neue Medien kompetent beurteilen und selbstverständlich nutzen, führt somit zu der weitreichenden Forderung, die Hochschule muss ihre Studienstruktur ändern. Sie muss, wenn das genannte Ziel als verbindlich akzeptiert wird, gewährleisten, dass der Einsatz neuer Medien in der Bildungsbiografie Lehramtsstudierender zu einem selbstverständlichen Element wird. Nur dann werden sie selbst als Lehrerinnen und Lehrer in ihrer Unterrichtsplanung auf neue Medien zurückgreifen. Gelingt dies nicht, so bleibt zu erwarten, dass während des Studiums angestellte Reflexionen zum Einsatz neuer Medien im Unterricht nicht die erhofften Früchte tragen, und die jun- 
gen Lehrerinnen und Lehrer statt dessen auf die ihnen aus der eigenen Erfahrung bekannten Unterrichtsmuster zurückgreifen werden (vgl. Blömeke 2004). Die Hochschullehrenden stehen hierbei vor demselben Problem wie die Lehrerinnen und Lehrer in der Schule, weshalb dem Fachbereich Medienpädagogik (oder Erziehungswissenschaften) zugeordnete medienpädagogische Beratungsstellen eingerichtet bzw. ausgebaut werden müssen. Diese Beratungsstellen haben die Aufgabe, die Lehrenden bei der schwierigen Aufgabe der Integration neuer Medien in ihre Lehre zu unterstützen. Nur wenn die Lehramtsstudierenden am Ende ihrer Ausbildung eine grundsätzlich positive Selbstwirksamkeitserwartung hinsichtlich ihrer Mediennutzungskompetenz und die Erfahrung der selbstverständlichen Integration neuer Medien in die Lehre mit in die Schule nehmen, besteht die Hoffnung, dass das eingangs genannte Ziel der Medienbildung als gesellschaftlicher Selbstzweck erreicht wird.

Zusammenfassend lassen sich fünf wesentliche Punkte festhalten, die das beschriebene Ziel der Förderung medienpädagogischer Handlungskompetenz Lehramtsstudierender intendieren. Diese sind:

1. Integration in die Bildungsbiografie: Sollen angehende Lehrerinnen und Lehrer in ihrem zukünftigen Berufsalltag neue Medien selbstverständlich einsetzen, so setzt dies voraus, dass sie den Umgang mit diesen in der eigenen Bildungsbiografie als bedeutsames Element erlebt haben. Elearning-Veranstaltungen dürfen daher kein Schattendasein an den Hochschulen fristen. Ferner dürfen sie nicht im Wesentlichen auf sich selbst referenzieren.

2. Verbindliche e-learning-Angebote in allen Fächern: Der Fachbereich Medienpädagogik kann dieser Aufgabe unmöglich im Alleingang gerecht werden. Es ist daher nötig, dass jeder Fachbereich dazu verpflichtet wird, einen verbindlichen Anteil seiner Lehrangebote als e-learningSeminare anzubieten. Ebenso müssen die Lehramtsstudierenden verpflichtet werden diese Angebote anzunehmen.

3. Förderung der Medienkompetenz: Der Fachbereich Medienpädagogik übernimmt in diesem Konzept dahingehend eine Schlüsselfunktion, dass er die nötigen auf Medienpädagogik bzw. Mediendidaktik referenzierenden Veranstaltungen anbieten soll. Lehramtsstudierende sollen neue Medien nicht nur als probate Unterrichtsmittel kennenlernen, sondern selbstverständlich auch Kriterien- bzw. Beurteilungskataloge und mögliche Szenarien kennenlernen, um entsprechende Angebote kompetent einsetzen oder verwerfen zu können.
4. Entwicklung von e-learning-Szenarien: Ferner soll der Fachbereich Medienpädagogik in Zusammenarbeit und auf Augenhöhe mit den einzelnen Fachdidaktiken medienpädagogische Szenarien für die Lehre entwickeln. Nur wenn die einzelnen Fachdisziplinen ihre Bedürfnisse medienpädagogisch umsetzen und dabei einen didaktischen Mehrwert erzielen können, lässt sich diese massive Umstrukturierung rechtfertigen.

5. Weiterbildungsangebote: Schließlich müssen umfassende Informations- und Weiterbildungsangebote für die Hochschullehrenden gemacht werden. Ohne entsprechende Angebote erscheint es wenig zielführend, elearning-Veranstaltungen vorzuschreiben. Hierfür müssen medienpädagogische Beratungsstellen eingerichtet und bestehende ausgebaut werden.

\section{Anmerkungen}

1 Der e-learning Begriff wird in diesem Artikel als Sammelbegriff verwendet. Gemeint sind stets on- und offline-Angebote in voll- oder teilvirtueller, mono- oder multimedialer, -codaler oder -modaler Ausprägung unabhängig von deren Interaktivitäts- oder Adaptivitätsgrad (vgl. Issing \& Klimsa 2002). Diese weite Definition erweist sich auf Grund der oben vorgeschlagenen getrennten Betrachtung von Medieneinsatz und Medienkompetenz als angebracht.

\section{Literatur}

Bundesministerium für Bildung und Forschung (BMBF) (2006): IT-Ausstattung der allgemeinbildenden und berufsbildenden Schulen in Deutschland. Bestandsaufnahme 2006 und Entwicklung 2001 bis 2006. Bonn, Berlin.

Blömeke, S. (2004): Empirische Befunde zur Wirksamkeit der Lehrerbildung. In: Blömeke, S., Reinhold, P., Tulodziecki, G., Wildt, J.: Handbuch Lehrerbildung. Bad Heilbrunn.

Issing, L. J. \& Klisma, P. (Hrsg.) (2002): Information und Lernen mit Multimedia und Internet. Lehrbuch für Studium und Praxis. Beltz, Weinheim 2002.

McLuhan, H. M. (1964): Understanding Media: The Extensions of Man.

Medienpädagogische Einrichtungen (2009): Keine Bildung ohne Medien. Medienpädagogisches Manifest. Magdeburg 2009.

(http://www.gmk-net.de/presse/090323.php 30.07.2009)

\section{Dipl.-Päd. Michael Lippstreu}

ist Doktorand im Promotionskolleg „ELearning in der Schule als Grundstein für lebenslanges Lernen“ an der PH Ludwigsburg. 